\title{
Local control of improper ferroelectric domains in $\mathrm{YMnO}_{3}$
}

\author{
Lukas Kuerten $\odot,{ }^{1,}{ }^{*}$ Stephan Krohns $\odot,{ }^{2}$ Peggy Schoenherr $\odot,{ }^{1,3}$ Katharina Holeczek, ${ }^{2}$ Ekaterina Pomjakushina, ${ }^{4}$ \\ Thomas Lottermoser, ${ }^{1}$ Morgan Trassin, ${ }^{1}$ Dennis Meier $\odot,{ }^{5}$ and Manfred Fiebig $\oplus^{1}$ \\ ${ }^{1}$ Department of Materials, ETH Zurich, 8093 Zurich, Switzerland \\ ${ }^{2}$ Experimental Physics V, University of Augsburg, 86135 Augsburg, Germany \\ ${ }^{3}$ School of Materials Science and Engineering, University of New South Wales, Sydney 2052, Australia \\ ${ }^{4}$ Laboratory for Multiscale Materials Experiments, Paul Scherrer Institut, 5232 Villigen, Switzerland \\ ${ }^{5}$ Department of Materials Science and Engineering, Norwegian University of Science and Technology NTNU, 7491 Trondheim, Norway
}

(Received 15 April 2020; revised 4 August 2020; accepted 6 August 2020; published 21 September 2020)

\begin{abstract}
Improper ferroelectrics are described by two order parameters: a primary one, driving a transition to long-range distortive, magnetic, or otherwise nonelectric order, and the electric polarization, which is induced by the primary order parameter as a secondary, complementary effect. Using low-temperature scanning probe microscopy, we show that improper ferroelectric domains in $\mathrm{YMnO}_{3}$ can be locally switched by electric field poling. However, subsequent temperature changes restore the as-grown domain structure as determined by the primary lattice distortion. The backswitching is explained by uncompensated bound charges occurring at the newly written domain walls due to the lack of mobile screening charges at low temperature. Thus, the polarization of improper ferroelectrics is in many ways subject to the same electrostatics as in their proper counterparts, yet complemented by additional functionalities arising from the primary order parameter. Tailoring the complex interplay between primary order parameter, polarization, and electrostatics is therefore likely to result in novel functionalities specific to improper ferroelectrics.
\end{abstract}

DOI: 10.1103/PhysRevB.102.094108

\section{INTRODUCTION}

In improper ferroelectrics, the spontaneous polarization emerges as subordinate effect to a primary order parameter which can be a lattice distortion, a magnetization, or another nonelectric quantity [1-4]. This dependence can lead to properties not observed in their polarization-driven proper ferroelectric counterparts. Improper ferroelectrics can be expected to be more robust towards extrinsic influences, such as depolarizing fields, allowing domain configurations with unusual head-to-head or tail-to-tail polarization geometries at the domain walls $[5,6]$. Such domain configurations can have technologically relevant properties, ranging from local conductance enhancement [7-9] to functionalities of advanced circuit elements $[10,11]$.

Both order parameters of improper ferroelectrics, the primary one and the induced polarization, can in principle influence the domain structure, but while it appears obvious that the primary order parameter sets the initial domain structure when crossing the transition temperature, the role played by the secondary order parameter and the associated electrostatics is not as clear. In the case of the hexagonal manganites $\left(R \mathrm{MnO}_{3}\right.$, with $\left.R=\mathrm{Sc}, \mathrm{Y}, \mathrm{In}, \mathrm{Dy}-\mathrm{Lu}\right)$, one of the most established classes of improper ferroelectrics, a lattice-trimerizing distortion as primary order parameter dominates the formation of domains, but only the secondary order parameter is susceptible to poling in an electric field [12]. Therefore, a key question is if, and how, domains formed in association with the emergence of the primary order parameter at the

*lukas.kuerten@mat.ethz.ch transition temperature $T_{\mathrm{c}}$ may differ from those created by electric field poling acting on the secondary order parameter within the ordered phase far below $T_{\mathrm{c}}$. In previous studies on the emergence and manipulation of ferroelectricity in the hexagonal manganites, the topology and the distribution of the resulting domains was considered [5,6,12], but not the material-specific dynamics of the poling process. Even though a detailed understanding of this issue is crucial for the functionalization of improper ferroelectrics, this aspect has not received much attention.

Here, we investigate electric-field poling at the nanometer scale in hexagonal $\mathrm{YMnO}_{3}$. In this material, uniform tilting of the $\mathrm{MnO}_{5}$ bipyramids in the unit cell and a concomitant shift of the yttrium ions occur at $1258 \mathrm{~K}$. This lattice-trimerizing distortive transition drives an improper ferroelectric polarization of $5.6 \mu \mathrm{C} \mathrm{cm}^{-2}$ along the hexagonal axis $[2,6,13-15]$. The resulting domain structure consists of six trimerizationpolarization domain states forming vortex-like meeting points with alternating polarization around the vortex core $[5,6,16]$.

We use atomic force microscopy (AFM) to apply local electric fields at cryogenic temperatures, where nonintrinsic effects due to barrier layer capacitances are negligible [17,18], creating polarization domains at the nanoscale. We compare these written domains to the domains formed via the primary order parameter at $T_{\mathrm{c}}$. We find that despite the secondary nature of the electric polarization, this polarization dominates the poling behavior just as in conventional ferroelectrics. Domains can be created at will by locally applied electric fields. Thermal annealing cycles, however, return the samples to the as-grown domain configuration. This recovery is explained 
by uncompensated bound charges at the domain walls and the surface, which arise due to decreasing availability of mobile carriers at cryogenic temperature. Hence, despite the secondary nature of the ferroelectric order, the electrostatic conditions overrule the primary lattice trimerization. Quite strikingly, we thus find that improper ferroelectrics retain key characteristics of proper ferroelectrics, yet complemented by functionalities introduced by the secondary nature of the electric order.

\section{METHODS}

Experiments were performed on $\mathrm{YMnO}_{3}$ single crystals grown by the floating-zone method [16,19]. The crystals were cut into platelets with a thickness of approximately $500 \mu \mathrm{m}$ perpendicular to the hexagonal axis. They were lapped with an $\mathrm{Al}_{2} \mathrm{O}_{3}$ solution and polished with silica slurry, yielding a surface roughness of approximately $1 \mathrm{~nm}$, suitable for AFM measurements. We thus obtained out-of-plane-polarized samples whose trimerization-polarization domains at the surface are separated by nominally uncharged $180^{\circ}$ side-by-side domain walls [5-7].

Dielectric measurements were performed using a Novocontrol Alpha analyzer (at $1 \mathrm{~Hz}$ to $1 \mathrm{MHz}$ ) and a TF2000 Aixacct system (hystersis loops, at $1 \mathrm{~Hz}$ ) in combination with a high-voltage booster for voltages up to $2 \mathrm{kV}$. Measurements were conducted at $50 \mathrm{~K}$ to $300 \mathrm{~K}$ in a closed-cycle refrigerator with samples in vacuum to avoid electrical discharge. The properties of semiconducting materials are often superimposed by extrinsic barrier layer contributions [20] which may affect polarization measurements $[21,22]$. For $\mathrm{YMnO}_{3}$, a temperature of $120 \mathrm{~K}$ and a frequency of $1 \mathrm{~Hz}$ avoid barrier layer capacitances and allow detecting the genuine ferroelectric properties of the material both in bulk and AFM experiments $[17,18]$.

AFM measurements were performed at $120 \mathrm{~K}$ to $250 \mathrm{~K}$ in an attoLiquid 2000 AFM setup (attocube GmbH, Germany) with ANSCM-PT Pt/Ir-coated conductive tips (AppNano Inc., USA) in two different modes: On one hand, we directly imaged the distribution of the polarization by piezoresponse force microscopy (PFM) [23-25]. In this mode, the AFM tip is brought into contact with the sample surface and an alternating voltage is applied to the tip. The instrument detects the contraction and expansion of the sample due to the piezoelectric effect. A contraction in phase or in antiphase with the excitation voltage corresponds to polarization in the upward or downward direction, respectively.

On the other hand, we used the presence of uncompensated charges on the surface to image the domain structure by electrostatic force microscopy (EFM) [26,27]. Due to a difference in strength of the pyroelectric effect, the surface charge differs between domains and domain walls, which is detected as contrast in EFM measurements. This contrast is further affected by structural and chemical defects, which show a propensity to accumulate at domain walls, locally enhancing the conductivity and thus reducing the surface charge. In our EFM experiment, the AFM tip is grounded so that mirror charges are induced on the tip by charges on the sample surface. In this way, the EFM measurement is sensitive to the density of surface charges, but not their polarity. For details of the EFM measurement, see Supplemental Material [28].

Local domain switching was achieved at $120 \mathrm{~K}$ by applying DC bias voltages to the AFM tip in contact with the sample surface while line scanning the surface at a constant speed of $2 \mu \mathrm{m} \mathrm{s}^{-1}$.

\section{RESULTS}

PFM measurements at $120 \mathrm{~K}$ showed the typical trimerization-induced improper ferroelectric domain structure of the hexagonal manganites [Fig. 1(a)]. We then created a new domain by applying a voltage of $+45 \mathrm{~V}$ to the AFM tip while scanning a window of $1 \mu \mathrm{m} \times 1 \mu \mathrm{m}$ [Fig. 1(b)]. This resulted in a square-shaped domain of upwards polarization (white arrow) within a down-polarized domain. The black arrow points to a region where the same poling procedure was applied to an area which was already polarized upwards. The latter led to the injection of surface charges, visible as a diffuse dark region because the surface charge screens the applied voltage and hence leads to a reduced piezoresponse, even though the intrinsic piezoelectric coefficient itself does not change. When a negative voltage of $-45 \mathrm{~V}$ is applied, the effects on up- and down-polarized domains are reversed (see Supplemental Material [28]). At $120 \mathrm{~K}$, the written domain was stable over a period of more than six days, whereas the surface charges disappeared within a few hours [Fig. 1(c)]. Finally, we found that when the sample was heated to $250 \mathrm{~K}$, the domain structure reverted to its original configuration, i.e., the electric-field-induced square domain disappeared [Fig. 1(d)].

In order to investigate how the ferroelectric domain structure reverts to its previous configuration, we recorded a series of PFM images at higher spatial resolution. Figure 2(a) shows a down-polarized as-grown bubble domain within an uppolarized environment. After scanning a window of $2 \mu \mathrm{m} \times$ $2 \mu \mathrm{m}$ covering the entire bubble with $+45 \mathrm{~V}$ applied to the tip, the polarization was mostly reversed so that the bubble disappeared. A faint outline, however, was still observable where the previous as-grown domain wall had been located [Fig. 2(b)]. This outline is possibly caused by the presence of oxygen interstitials, which are known to accumulate at neutral walls [11] but are effectively immobile at low temperature [29] and hence cannot follow the displacement of the domain wall. These defects are visible in the PFM measurement, because they cause a local difference in Schottky barrier and hence change the effective applied voltage. When the sample was heated to $250 \mathrm{~K}$, the original domain structure was recovered as depicted in Fig. 2(c). A minor change in overall contrast in the poled region is due to the removal of residual dirt from the surface by scanning with high load and applied voltage.

Complementary to the local measurements, we performed bulk dielectric spectroscopy and ferroelectric-hysteresis-loop measurements to characterize the retention of the $\mathrm{YMnO}_{3}$ polarization and test for signatures of backswitching at the macroscale. Measurements of the dielectric constant $\epsilon^{\prime}$ shown in Fig. 3(a) revealed a steplike increase of $\epsilon^{\prime}$ with temperature, indicating an intrinsic dielectric constant masked by barrierlayer capacitance effects [20,30]. Therefore, we chose our measurement temperature such that we could probe the in- 


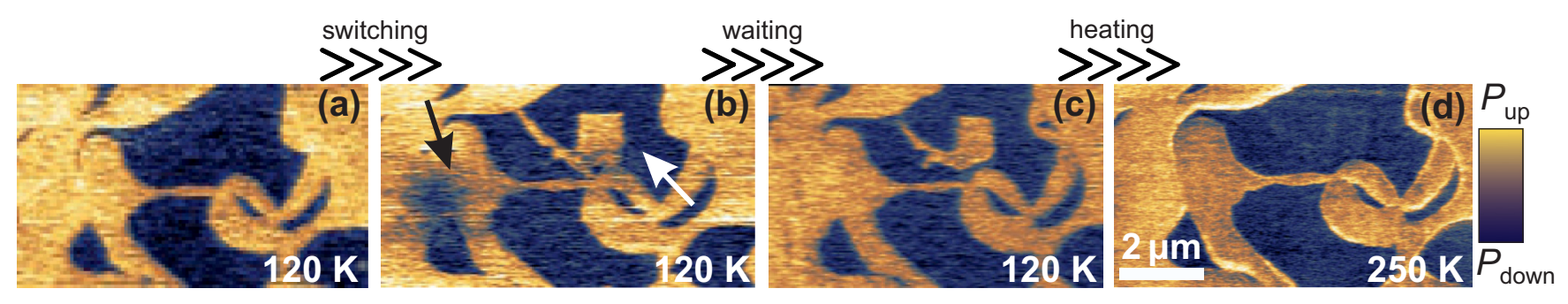

FIG. 1. Creation of improper ferroelectric domains in $\mathrm{YMnO}_{3}$ by local cryogenic-temperature electric-field poling. (a) Pristine domain structure measured by PFM at $120 \mathrm{~K}$. (b) A square-shaped area (white arrow) of reversed polarization is created by scanning while applying $+45 \mathrm{~V}$ to the AFM tip in contact with the surface. The bright line protruding from the lower end of the square was caused by moving the AFM tip into position for the poling with the poling voltage applied. When the same voltage is applied to an area polarized in the direction of the applied voltage, surface charging results in a diffuse change of contrast (black arrow). (c) The same area of the sample surface imaged after remaining at $120 \mathrm{~K}$ for 6 days after the poling. The artificially created domain is still present, whereas the surface-charged area has disappeared. (d) At $250 \mathrm{~K}$ the domain structure abruptly reverts to the original configuration in (a). A minor variation in contrast between panels (a)-(d) is due to differences in scan speed and tip wear over the duration of six days.

trinsic ferroelectric polarization $[17,18,20]$ [left of the dashed lines in Fig. 3(a)]. Specifically, we performed all experiments at or below $140 \mathrm{~K}$. For confirmation, we measured a ferroelectric hysteresis loop at $120 \mathrm{~K}$ [inset of Fig. 3(b)]. The shape of the loop and the saturation polarization are in agreement with theory [15] and values of previous experiments [5,12,18,31], confirming that only the true polarization was measured.

To measure the retention behavior, first a pre-poling pulse with an applied electric field of $120 \mathrm{kV} \mathrm{cm}^{-1}$ was used to saturate the sample polarization. After a delay time ranging from $1 \mathrm{~s}$ to $3.6 \times 10^{-5} \mathrm{~s}$, positive-up-negative-down (PUND) measurements with the first pulse in the same electric-field direction as the pre-poling pulse and a peak electric field of $120 \mathrm{kV} \mathrm{cm}^{-1}$ were performed. From these, we determined the remaining fraction of the saturated polarization $p_{\mathrm{r}}(t)=$ $P_{\text {meas }}(t) / P_{\text {sat }}$, where $P_{\text {sat }}$ denotes the initial polarization created by the pre-poling pulse and $P_{\text {meas }}$ the measured polarization after the delay time $t$. Figure 3(b) shows $p_{\mathrm{r}}$ as a function of the delay time $t$ measured at three different temperatures. The equilibrium state towards which the system relaxes corresponds to $p_{\mathrm{r}}=50 \%$, i.e., an equal fraction of up- and down polarized regions. At $140 \mathrm{~K}$, the polarization reverted quickly to this equilibrium state after poling, whereas at $120 \mathrm{~K}$, the value of polarization surplus was retained for several days. Despite this stability, however, minor relaxation effects in the domain structure were observed even at $120 \mathrm{~K}$. These results are consistent with the local switching experiments in Figs. 1 and 2 , showing stability of the electric-field induced domain structure at $120 \mathrm{~K}$ and fast relaxation at higher temperatures.

The domain walls of as-grown and electric-field-induced polar domains also showed different behavior when observed in EFM measurements. Because the overall conductivity is very low at $120 \mathrm{~K}$, the domain wall conductance cannot be measured directly by conductive AFM. However, EFM allows us to image the electrostatics of domain walls even under insulating conditions (see Ref. [27] and Supplemental Material [28]). Figure 4(a) shows a PFM scan of the sample surface where a surface domain was created by poling at $120 \mathrm{~K}$ (arrow). Here, in contrast to the measurements in which the AFM tip was scanned over a defined area with an applied voltage, the tip was stationary on the sample surface while applying the writing voltage. This resulted in the creation of a domain of about $300 \mu \mathrm{m}$ diameter as shown in Fig. 4(a).

Figure 4(b) shows an EFM image of the same area, measured at $120 \mathrm{~K}$ after the sample had been heated to $200 \mathrm{~K}$. This temperature sequence creates an EFM contrast due to the pyroelectric effect associated with the temperature change but preserves the written domain pattern because the temperature is not high enough for fast relaxation (see Supplemental Material [28] for details). A pronounced EFM contrast was observed at as-grown domain walls, which is consistent with their enhanced conductivity attributed to the presence of oxy-
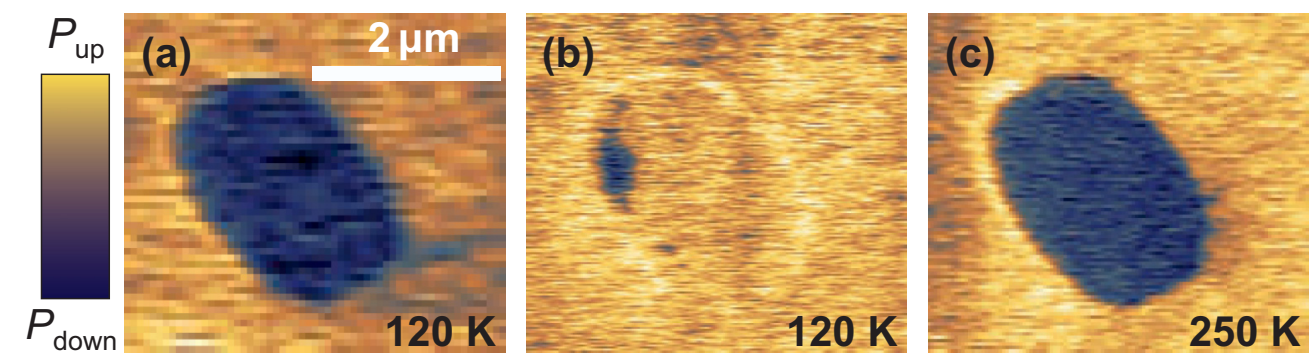

FIG. 2. Elimination of improper ferroelectric domains in $\mathrm{YMnO}_{3}$ by local low-temperature electric-field poling. (a) A down-polarized as-grown bubble domain in an up-polarized environment measured by PFM at $120 \mathrm{~K}$. (b) The polarization of the bubble domain is reversed by scanning a window of $2 \mu \mathrm{m} \times 2 \mu \mathrm{m}$ covering the bubble with $+45 \mathrm{~V}$ applied to the AFM tip. Note that the outline of the original domain is still visible in the PFM image. (c) When increasing the temperature to $250 \mathrm{~K}$, the original bubble domain in (a) is reestablished. 

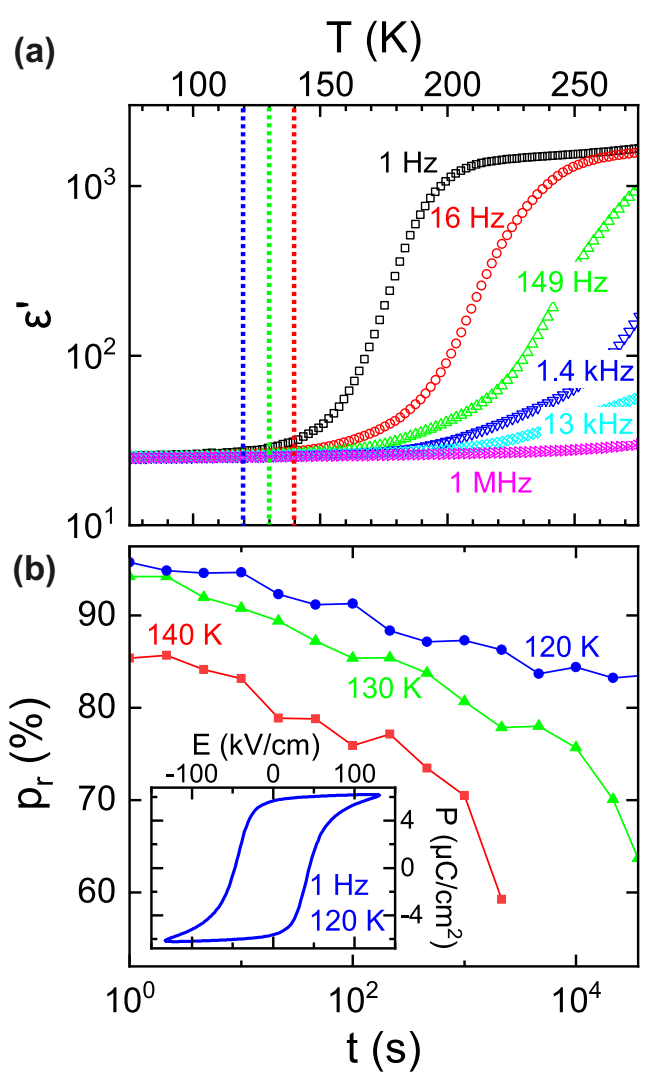

FIG. 3. Spatially integrated bulk ferroelectric properties of $\mathrm{YMnO}_{3}$. (a) Temperature-dependent dielectric constant for selected frequencies measured by dielectric spectroscopy. The dashed lines denote the temperatures below which the intrinsic ferroelectric properties of the sample can be measured. (b) Time-dependent decay of the saturated polarization $\left[p_{\mathrm{r}}(t)=P_{\text {meas }}(t) / P_{\text {sat }}\right.$, see text]. At $120 \mathrm{~K}$, the polarization is retained for several days, whereas at $140 \mathrm{~K} p_{\mathrm{r}}(t)$ relaxes towards equilibrium, i.e., $p_{\mathrm{r}}=50 \%$, within a few hours. Inset: ferroelectric hysteresis loop measured at $120 \mathrm{~K}$ and $1 \mathrm{~Hz}$.

gen interstitials [11]. At the domain walls associated with the written domains, however, the EFM contrast was weaker by a factor of 2.5 on average (as obtained from a number of representative line profiles), suggesting lower electronic conductance and, hence, a lower density of oxygen defects compared to the as-grown walls. In the PFM measurements, however, the as-grown and the electric-field-induced domains exhibited the same brightness.

\section{DISCUSSION}

We now discuss why electric-field-induced domains tend to return to their as-grown, trimerization-controlled configuration upon heating. We emphasize that even though the electric-field poling acts on the polarization, the trimerization has to follow this reorientation because of the rigid coupling between secondary and primary order parameter [32]. Thus, we can exclude that the observed backswitching is due to an unswitched residue of the trimerized state. In addition, in all PFM measurements, electric-field induced and asgrown domains showed the same domain contrast, and in bulk measurements electric-field poling yields the expected
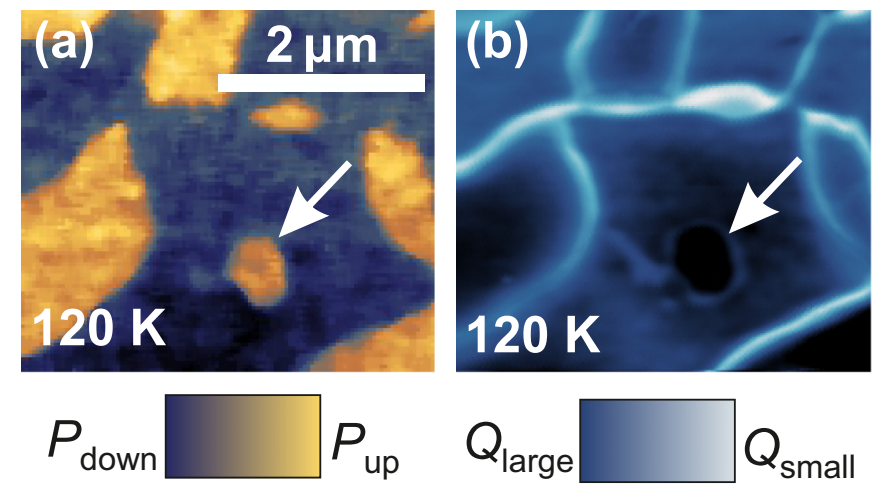

FIG. 4. Electrostatic contrast at as-grown and electric-fieldinduced domain walls at $120 \mathrm{~K}$. (a) PFM scan of the sample surface. An electric-field-induced surface domain is highlighted by the white arrow. (b) EFM scan measuring the surface charge $Q$ of the same area as in (a). Even though the PFM contrast is the same for both asgrown and electric-field-induced domains, the EFM contrast of the respective domain walls differs strongly between the two differently generated domains. Some domains which appear disconnected in the PFM image are in fact connected by channel-like domains below the resolution limit. This can be seen in the EFM image where the topological domain structure becomes evident.

saturation polarization. Our measurements are thus consistent with electric-field-induced and as-grown domains exhibiting the same properties, specifically the same polarization.

When the polarization at the sample surface is locally influenced by the AFM tip, it is affected only to a depth of a few hundreds of nanometers away from the surface due to field focusing below the AFM tip. Hence, the bulk polarization below the field-induced square domain in Fig. 1 remained unswitched. At the newly created buried domain wall below, the polarizations meet in a tail-to-tail configuration, resulting in uncompensated charges as is illustrated in Fig. 5(a). At elevated temperatures, such a configuration would be readily screened by charge carriers, but in the cryogenic environment of our experiment, this screening process becomes slower than the timescale of the experiment. Since the presence of uncompensated bound charges at the domain wall is energetically unfavorable, the material returned to its initial configuration when heated.
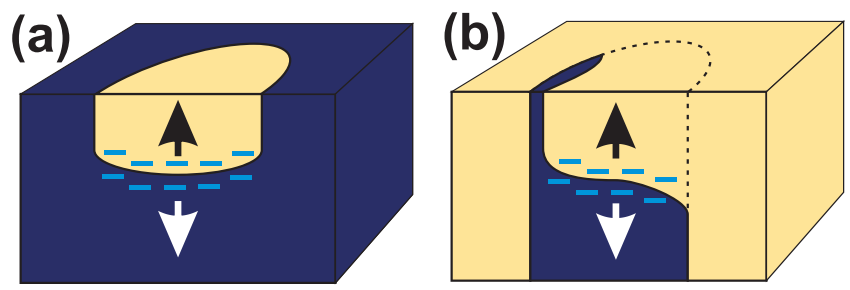

FIG. 5. Schematic cross section of tip-electric-field-induced domain configurations and distribution of uncompensated charges $(-)$. Arrows denote the polarization direction of the respective domains. (a) Creation of a new domain at the surface, as described in Fig. 1. (b) Deleting an as-grown bubble domain from the surface, as described in Fig. 2. Vertical dimensions not to scale. 
As-grown domains, on the other hand, exhibit no uncompensated charges and are therefore stable. An as-grown domain wall which had been erased by external electric fields was restored to its original shape by a temperature increase (Fig. 2). We conclude that defects, which show a propensity to accumulate at domain walls at room temperature, but are mostly immobile at cryogenic temperatures [29], could remain at their original location when a domain wall is displaced and serve as localized potential energy minima for the recovery of the domain structure. This hypothesis is corroborated by the remanent outline of the erased domain in Fig. 2(b) and the difference in domain wall contrast between as-grown and electric-field-induced domain walls in Fig. 4(b), which can both be explained by a difference in defect density. A minor migration of defects to domain walls may occur even at cryogenic temperatures, which explains the very faint outline of the previously poled region observed in Fig. 1(d). Note that a similar dissociation of domain walls and defects during switching was previously found in $\mathrm{BiFeO}_{3}$ [33]. The intriguing consequence of this mechanism is that the electrostatic forces in the improper ferroelectric $\mathrm{YMnO}_{3}$ are strong enough to reverse not only the secondary, but also the primary order parameter, leading to the striking situation that the allegedly weaker order parameter controls the stronger one.

Note that in all our local probe experiments, the topological protection of the domain structure by the primary order parameter did not play a role, because only domains within existing domains at the surface were created and erased, whereas the topological domain-vortex meeting points were not affected. Therefore, we observed a behavior resembling that of proper ferroelectrics.

In our bulk switching experiments, on the other hand, the topological constraints imposed by the primary order parameter affected the poling behavior. Specifically, electricfield poling cannot destroy the topological domain vortices and therefore the sample cannot be transferred into a singledomain state $[5,6]$. These unswitched remnants of the unfavored polarization direction then served as nuclei and memory in the relaxation of the polarization, a behavior not observed in proper ferroelectrics.

\section{SUMMARY AND CONCLUSIONS}

We have shown that despite their origin in a nonpolar, primary order parameter, the manipulation of polar domains in improper ferroelectric $\mathrm{YMnO}_{3}$ is guided by the same electrostatics as in proper ferroelectrics. In particular, the improper ferroelectric domain configuration can be manipulated by electric fields, and its dynamics upon heating is driven by the migration of electric charges to domain walls. On the other hand, bulk measurements indicate that the topological protection of the domain configuration due to the primary order parameter prevents the sample from reaching the single-domain configuration of a proper ferroelectric, with consequences for the nucleation, pinning, and conductance of the remaining ferroelectric domain walls. We thus conclude that with regard to external fields and charges, improper ferroelectrics behave like a proper ferroelectric in many respects, but the existence of the primary order parameter leads to intriguing additional functionalities.

\section{ACKNOWLEDGMENTS}

The authors thank M. C. Weber for valuable help in sample preparation and A. Bortis, D. M. Evans, and Q. N. Meier for helpful discussions. This research was supported by the EU European Research Council (Advanced Grant No. 694955INSEETO) and the Swiss National Science Foundation under Grant Nos. SNSF 20021_178825, 20021_149192, and 206021_150635. L.K. acknowledges support from an ETH Career Seed Grant. K.H. \& S.K. acknowledge funding from the Deutsche Forschungsgemeinschaft via the Transregional Collaborative Research Center TRR 80. D.M. thanks NTNU for support through the Onsager Fellowship Program and the Outstanding Academic Fellows Program.

All authors discussed the results and contributed to the completion of the paper. L.K. and P.S. performed the lowtemperature AFM experiments. S.K. and K.H. performed the dielectric spectroscopy measurements. E.P. grew the $\mathrm{YMnO}_{3}$ samples. L.K., S.K., T.L, M.T., D.M., and M.F. designed the experiment and supervised the study.
[1] A. P. Levanyuk and D. G. Sannikov, Sov. Phys. Usp. 17, 199 (1974).

[2] T. Kimura, T. Goto, H. Shintani, K. Ishizaka, T. Arima, and Y. Tokura, Nature (London) 426, 55 (2003).

[3] B. B. V. Aken, T. T. Palstra, A. Filippetti, and N. A. Spaldin, Nat. Mater. 3, 164 (2004).

[4] N. Ikeda, H. Ohsumi, K. Ohwada, K. Ishii, T. Inami, K. Kakurai, Y. Murakami, K. Yoshii, S. Mori, Y. Horibe, and H. Kitô, Nature (London) 436, 1136 (2005).

[5] T. Choi, Y. Horibe, H. T. Yi, Y. J. Choi, W. Wu, and S.-W. Cheong, Nat. Mater. 9, 253 (2010).

[6] T. Jungk, Á. Hoffmann, M. Fiebig, and E. Soergel, Appl. Phys. Lett. 97, 012904 (2010).

[7] D. Meier, J. Seidel, A. Cano, K. Delaney, Y. Kumagai, M. Mostovoy, N. A. Spaldin, R. Ramesh, and M. Fiebig, Nat. Mater. 11, 284 (2012).
[8] Y. S. Oh, X. Luo, F.-T. Huang, Y. Wang, and S.-W. Cheong, Nat. Mater. 14, 407 (2015).

[9] R. G. McQuaid, M. P. Campbell, R. W. Whatmore, A. Kumar, and J. M. Gregg, Nat. Commun. 8, 15105 (2017).

[10] J. A. Mundy, J. Schaab, Y. Kumagai, A. Cano, M. Stengel, I. P. Krug, D. M. Gottlob, H. Doğanay, M. E. Holtz, R. Held, Z. Yan, E. Bourret, C. M. Schneider, D. G. Schlom, D. A. Muller, R. Ramesh, N. A. Spaldin, and D. Meier, Nat. Mater. 16, 622 (2017).

[11] J. Schaab, S. H. Skjærvø, S. Krohns, X. Dai, M. E. Holtz, A. Cano, M. Lilienblum, Z. Yan, E. Bourret, D. A. Muller, M. Fiebig, S. M. Selbach, and D. Meier, Nat. Nanotechnol. 13, 1028 (2018).

[12] M. Lilienblum, T. Lottermoser, S. Manz, S. M. Selbach, A. Cano, and M. Fiebig, Nat. Phys. 11, 1070 (2015). 
[13] M. Fiebig, T. Lottermoser, D. Fröhlich, A. V. Goltsev, and R. V. Pisarev, Nature (London) 419, 818 (2002).

[14] T. Lottermoser, M. Fiebig, and D. Fröhlich, J. Appl. Phys. 91, 8251 (2002).

[15] C. J. Fennie and K. M. Rabe, Phys. Rev. B 72, 100103(R) (2005).

[16] Q. N. Meier, M. Lilienblum, S. M. Griffin, K. Conder, E. Pomjakushina, Z. Yan, E. Bourret, D. Meier, F. Lichtenberg, E. K. H. Salje, N. A. Spaldin, M. Fiebig, and A. Cano, Phys. Rev. X 7, 041014 (2017).

[17] E. Ruff, S. Krohns, M. Lilienblum, D. Meier, M. Fiebig, P. Lunkenheimer, and A. Loidl, Phys. Rev. Lett. 118, 036803 (2017).

[18] A. Ruff, Z. Li, A. Loidl, J. Schaab, M. Fiebig, A. Cano, Z. Yan, E. Bourret, J. Glaum, D. Meier, and S. Krohns, Appl. Phys. Lett. 112, 182908 (2018).

[19] B. Roessli, S. N. Gvasaliya, E. Pomjakushina, and K. Conder, J. Exp. Theor. Phys. Lett. 81, 287 (2005).

[20] P. Lunkenheimer, S. Krohns, S. Riegg, S. Ebbinghaus, A. Reller, and A. Loidl, Eur. Phys. J.: Spec. Top. 180, 61 (2009).

[21] J. F. Scott, J. Phys.: Condens. Matter 20, 021001 (2007).

[22] A. Loidl, S. Krohns, J. Hemberger, and P. Lunkenheimer, J. Phys.: Condens. Matter 20, 191001 (2008).
[23] S. Kalinin and A. Gruverman, (eds.), Scanning Probe Microscopy (Springer, New York, 2007).

[24] E. Soergel, J. Phys. D: Appl. Phys. 44, 464003 (2011).

[25] A. Gruverman, M. Alexe, and D. Meier, Nat. Commun. 10, 1661 (2019).

[26] S. V. Kalinin and D. A. Bonnell, Phys. Rev. B 63, 125411 (2001).

[27] P. Schoenherr, K. Shapovalov, J. Schaab, Z. Yan, E. D. Bourret, M. Hentschel, M. Stengel, M. Fiebig, A. Cano, and D. Meier, Nano Lett. 19, 1659 (2019).

[28] See Supplemental Material at http://link.aps.org/supplemental/ 10.1103/PhysRevB.102.094108 for additional information on the imaging of domain walls by electrostatic force microscopy, effects of reversed poling voltage, and PFM measurement channels.

[29] S. Remsen and B. Dabrowski, Chem. Mater. 23, 3818 (2011).

[30] P. Lunkenheimer, V. Bobnar, A. V. Pronin, A. I. Ritus, A. A. Volkov, and A. Loidl, Phys. Rev. B 66, 052105 (2002).

[31] M.-G. Han, Y. Zhu, L. Wu, T. Aoki, V. Volkov, X. Wang, S. C. Chae, Y. S. Oh, and S.-W. Cheong, Adv. Mater. 25, 2415 (2013).

[32] Y. Kumagai and N. A. Spaldin, Nat. Commun. 4, 1540 (2013).

[33] I. Stolichnov, M. Iwanowska, E. Colla, B. Ziegler, I. Gaponenko, P. Paruch, M. Huijben, G. Rijnders, and N. Setter, Appl. Phys. Lett. 104, 132902 (2014). 